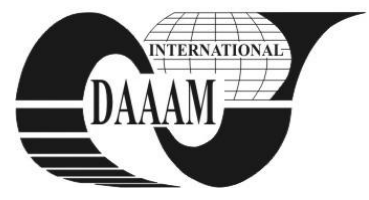

\title{
ADAPTIVE PREDICTIVE CONTROL OF NONLINEAR SERVO SYSTEM
}

\section{PIKNER, M[ichal] \& KUBALCIK, M[arek]}

\begin{abstract}
This paper deals with design and implementation of self-tuning predictive control for control of a real servo - motor system. The control algorithm was realized as a self - tuning controller with the recursive least square method in the identification part. The initial parameter estimates were set on the basis of the transfer function of the system. The predictive controller considers constraints of input variables. The algorithm was tested on a non-linear real system AMIRA DR300
\end{abstract}

Key words: self-tuning, GPC, constrains, least squares method, laboratory model AMIRA DR300

\section{INTRODUCTION}

Model Based Predictive Control (MBPC) or only Predictive Control is one of the control methods which have developed considerably over a few past years. Predictive control is essentially based on discrete or sampled models of processes. Computation of appropriate control algorithms is then realized namely in the discrete domain.

\section{PREDICTIVE CONTROL}

The term Model Predictive Control presents a class of control methods which have common particular attributes (Camacho \& Bordons, 2004; Mikleš \& Fikar, 2007; Bobál et al., 2009; Bobál, 2009; Rossiter, 2003):

- Mathematical model of a system control is used for prediction of future control of a system output.

- The input reference trajectory in the future is known.

- A computation of the future control sequence includes minimization of an appropriate objective function (usually quadratic one) with the future trajectories of control increments and control errors.

- Only the first element of the control sequence is applied and the whole procedure of the objective fiction minimization is repeated in the next sampling period. The principle of Model Based Predictive Control (MBPC) is shown in Fig. 1, where is the manipulated variable, is the process output and is the reference signal, and are called minimum, maximum and control horizont. This principle is presented in Fig. 1.

\subsection{Generalized Predictive Control}

The output predictions of the Generalized Predictive Controller are based on using a CARIMA model:

$$
A\left(z^{-1}\right) y(t)=B\left(z^{-1}\right) z^{-d} u(t-1)+C\left(z^{-1}\right) \frac{e(t)}{\Delta}
$$

Where the unmeasurable disturbance is given by a white noise coloured by $C\left(z^{-1}\right)$. GPC uses a quadratic cost function of the form:

$$
\begin{aligned}
J\left(N_{1} N_{2} N_{u}\right) & =\sum_{j=N_{1}}^{N_{2}} \delta(j)[\hat{y}(t+j \mid t)-w(t+j)]^{2}+ \\
& +\sum_{j=N_{1}}^{N_{2}} \lambda(j)[\Delta \mathrm{u}(\mathrm{t}+\mathrm{j}-1)]
\end{aligned}
$$

Prediction can be written as

$$
\hat{y}=G u+f
$$

where $G$ is the matrix of the step response and $f$ is the free response.

Control algorithm has the form:

$$
\begin{gathered}
u=\left(G^{T} G+\lambda I\right)^{-1} G^{T}(w-f) \\
\Delta u=K(w-f)
\end{gathered}
$$

where $\mathrm{K}$ is first row of matrix $\left(G^{T} G+\lambda I\right)^{-1} G^{T}$.

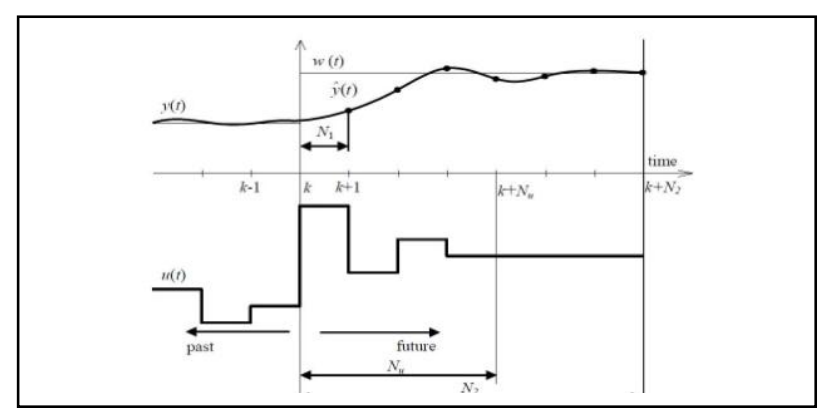

Fig. 1. The basic principle of the predictive control

\subsection{Constrained Model Predictive Control}

General formulation of predictive control with constraints is as follows

$$
\min _{\Delta u} 2 g^{T} \Delta u+\Delta u^{T} H \Delta u
$$

owing to

$$
A \Delta u \leq b
$$

$$
A=\left[\begin{array}{c}
I_{N \times N} \\
-I_{N \times N} \\
T \\
-T \\
G \\
-G
\end{array}\right] \quad b=\left[\begin{array}{c}
l \bar{u} \\
-l \underline{u} \\
l \bar{U}-l u(t-1) \\
-l \underline{U}+l u(t-1) T \\
l \bar{y}-f \\
-l y+f
\end{array}\right]
$$

\section{SYSTEM IDENTIFICATION}

Recursive least squares algorithm (Corriou, 2004) has the general form 


$$
\hat{\Theta}(k)=\hat{\Theta}(k-1)+K(k)\left[y(k)-\hat{\Theta}^{T}(k-1) \Phi(k)\right]
$$

where

$$
\widehat{\Theta}^{\mathrm{T}}(k)=\left[\begin{array}{llll}
a_{1} & a_{2} & b_{1} & b_{2}
\end{array}\right]
$$

is the vector of the parameters and

$$
\Phi(k)=[-y(k-1)-y(k-2) u(k-1) u(k-2)]
$$

Time-variable vector amplification (correction factor) is given by

$$
K(k)=\frac{C(k-1) \Phi(k)}{1+\Phi^{T}(k) C(k-1) \Phi(k)}
$$

And covariance matrix is

$$
C(k)=C(k-1)\left[1-\frac{\Phi(k) \Phi^{T}(k) C(k-1)}{1+\Phi(k) C(k-1) \Phi(k)}\right]
$$

\section{DESCRIPTION OF LABORATORY MODEL}

Laboratory model Amira DR300 (see Fig. 2) contains the technical realization of a nonlinear single-input/single-output system consisting of drive motor (M1), identical motor (M2) (used as current generator), tachogenerator $(\mathrm{T})$, incremental encoder (I) and clutch (K). A block scheme of the Amira DR300 is presented in Fig. 3.

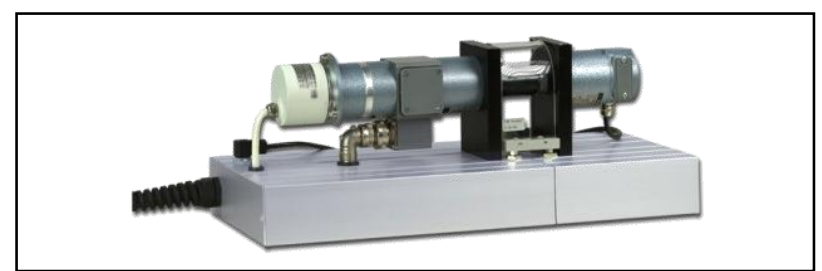

Fig. 2. Laboratory model Amira DR300

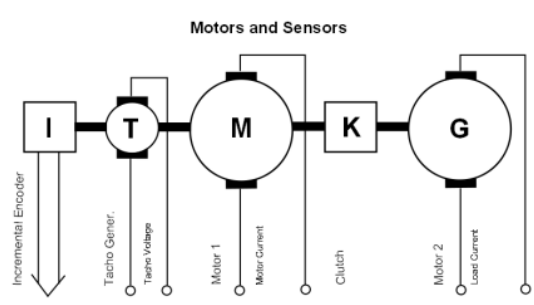

Fig. 3. Block scheme of Amira DR300

\section{EXPERIMENTAL RESULTS}

The Amira DR300 is a non-linear system. Its non-linear static characteristic is shown in Fig. 4.

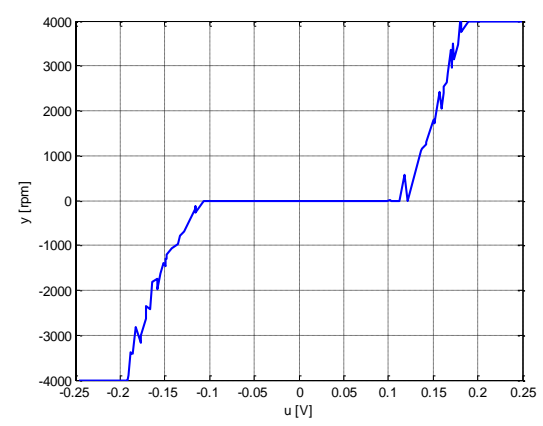

Fig. 4. Non-linear static characteristic
The initial parametres of recursive identification were determined from the transfer function (14). This transfer function was determined from linear part of the static characteristic.

$$
G(z)=\frac{B\left(z^{-1}\right)}{A\left(z^{-1}\right)}=\frac{0.2517 z^{-1}-0.022 z^{-2}}{1-0.9293 z^{-1}+0.147 z^{-2}}
$$

Sample time period $T_{0}=0.05 \mathrm{~s}$ was used for identification and control of system. Recussive identification was realized by the least squared method. Its results are presented in Fig. 5.

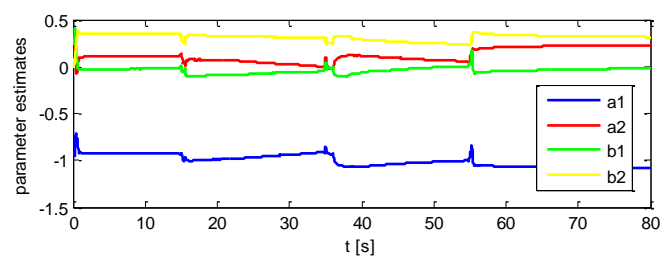

Fig. 5. Courses of parameter estimates

Minimal, maximal and control horizonts of predictive control were $N_{1}=1, N_{2}=5, N_{u}=3$. The penalty constant was $\lambda=5$. Results of self-tuning predictive control are in Fig. 6 .
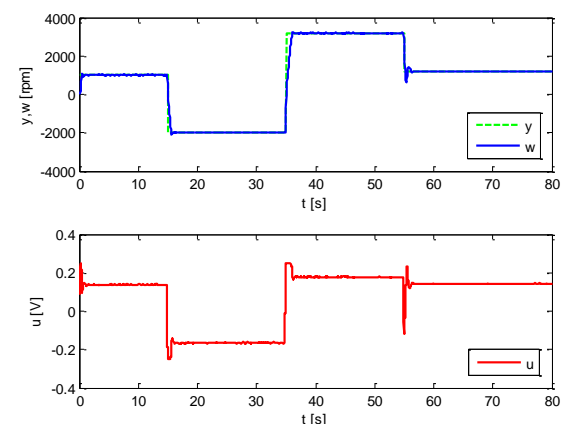

Fig. 6. Control with enhanced adaptation

\section{CONCLUSION}

The control tests executed on the laboratory model gave satisfactory results. The objective laboratory model simulates a process, which frequently occur in industry. It was proved that the examined method could be implemented and used successfully to control such processes.

\section{ACKNOWLEDGEMENTS}

This work was supported in Tomas Bata University in Zlin under grant IGA/34/FAI/11/D.

\section{REFERENCES}

Bobál, V. (2008). Adaptive and Predictive control, Tomas Bata University in Zlín, Academia centre, 978-807318-662-3, Zlín (in Czech)

Balate, J. (2003). Automticke rizeni, 1. release, BEN, 80-7300020-2,Prague

Camacho, E. F. \& Bordons, C. (2004). Model Predictive Control, Springer-Verlag, 978-1-85233-694-3, London

Rossiter, J. A. (2003). Model-Based Predictive Control A Practical approach,CRC Press, 0-8493-1291-4,USA

Corriou, J. P. (2004). Process control Theory and Applications, 1. Release, London: Springer-Verlag, 1-85233-776-1, London 